\title{
A Case of Ruptured Penile Epidermal Cyst
}

\section{Kyu Hyoung Cho}

Department of Urology, Soonchunhyang University Gumi Hospital, Soonchunhyang University College of Medicine, Gumi, Korea

\begin{abstract}
Epidermal cyst, a benign tumor, can occur after trauma and surgical interventions such as implanting epidermal keratinized squamous epithelial cells and sebaceous glands into the dermis and subcutaneous tissues. It can occur anywhere in the body. However, penile epidermal cysts are very rare. We present a case of a symptomatic penile mass of a 34-year-old male patient with a ruptured epidermal cyst.
\end{abstract}

Keywords: Epidermal cyst; Penis; Rupture; Case report

\section{INTRODUCTION}

The epidermal cyst can occur after implanting epidermal keratinized squamous epithelial cells and sebaceous glands into the dermis and subcutaneous tissues [1]. It is a benign lesion that can develop in any part of the body. Although a cutaneous epidermal cyst is a common lesion, penile localization is quite rare [2]. Most such cysts are asymptomatic. However, if these lesions grow large enough to disturb the normal function of the surrounding structures, become infected, or rupture into adjacent soft tissues, they may become symptomatic [3]. In this study, we report a huge, firm, and tender penile mass in a 34-year-old man. It was diagnosed as a ruptured epidermal cyst.

\section{CASE REPORT}

A 34-year-old male visited the hospital with a painful penile mass. He stated that the mass had been slowly increasing 20 years ago and that the pain started a few days ago for no apparent reason before he visited the hospital. The patient had a circumcision 20 years ago. Since then, he noticed that there was a mass. However, he did not seek any treatment because the mass was small and asymptomatic. He had no history of trauma, inflammation, urinary tract infection, hematuria, or dysuria. In physical examination, the mass was located at the ventral surface of the penile distal shaft. It was round, firm, tender, and freely movable within the dermis (Fig. 1). It had a smooth surface. Penile ultrasonography demonstrated a $5 \times 4 \times 3 \mathrm{~cm}$ slightly increased echoic cystic lesion in the subcutaneous layer of the penis (Fig. 2). There was no relation between the cystic lesion and cavernous bodies. Excision of the mass was performed under general anesthesia. With a transverse incision on the ventral aspect of the penis, we performed gentle dissection around the mass. The capsule of the mass was preserved and enucleated from surrounding tissues (Fig. 3). Histologic examination revealed that the capsule wall was lined with stratified squamous epithelium. A focal rupture site was confirmed in a specific part of the wall. There were more macrophages and lymphocytes compared to other tissues. The cavity contained keratinized materials inside (Fig. 4). The final diagnosis was a ruptured epidermal cyst of the penis. No recurrence has been noted for one year since the excision.

The patient provided written informed consent for the publication of clinical details and images.

\section{DISCUSSION}

Epidermal cysts are also known as epidermoid cysts. They are common benign tumors presenting anywhere in the body. Most 

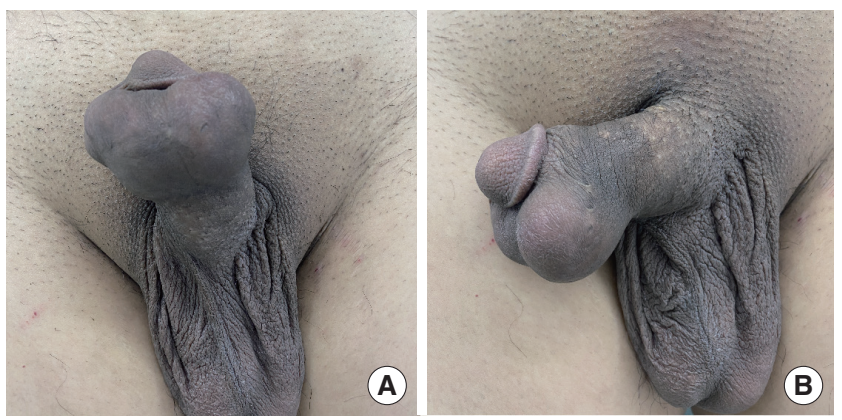

Fig. 1. (A) A preoperative photograph showing redness around the orifice. (B) A photograph of the extracted stones.

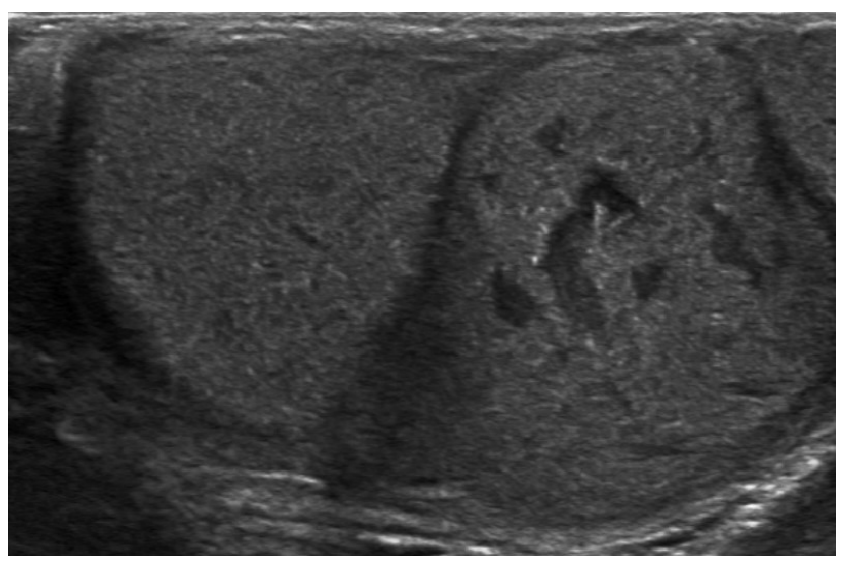

Fig. 2. Penile ultrasonography showed a $5 \times 4 \times 3 \mathrm{~cm}$ slightly increased echoic cystic lesion.

cysts remain asymptomatic. However, if these lesions grow large enough to disturb the normal function of surrounding structures, become infected, or rupture into adjacent soft tissues, they might become symptomatic [3]. The patient in this case had sudden onset of pain. The epidermal cyst might be formed by several mechanisms. They may result from implantation and proliferation of epidermal elements into the dermis and surgical implantation of epidermal tissues. They may also arise from the sequestration of epidermal rests during embryonic life, occlusion of the pilosebaceous unit, or traumatic implantation of epithelial elements [2]. Its differential diagnoses include dermoid cyst, teratoma, urethral diverticulum, glomus tumor, pilonidal cyst and epidermal cyst, and steatocystoma [4]. Epidermal cysts contain keratinized materials surrounded by keratinized squamous epithelial cells. They can be congenital or acquired. Abnormal embryologic closure of the median raphe has been postulated to represent the congenital form of a penile epidermal cyst [1]. Acquired epidermal cysts can occur because of the occluded pilosebaceous unit or traumatic/mechani-
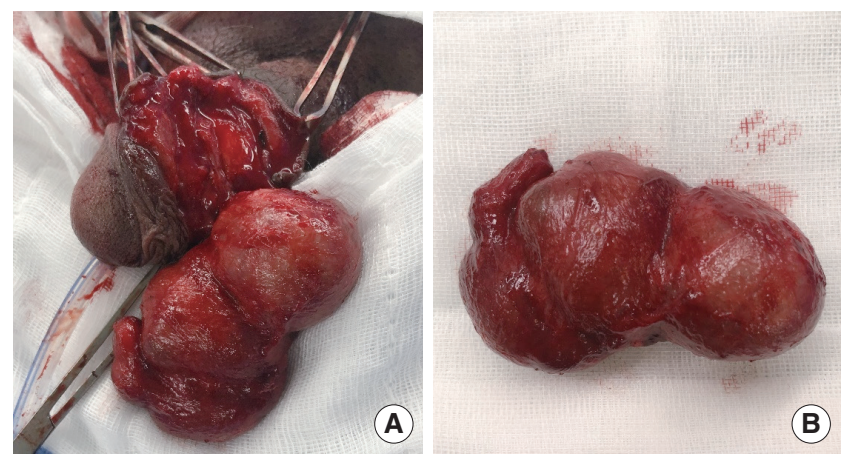

Fig. 3. (A) Operative view of the mass during excision. (B) Excised specimen.

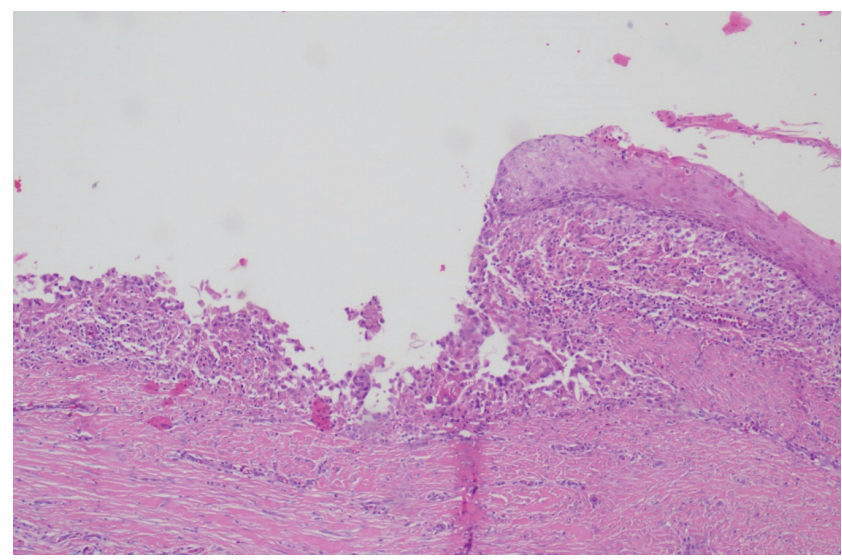

Fig. 4. Histologic aspect of the ruptured cyst wall (H\&E, $\times 100)$.

cal implantations involving the implantation of epithelial elements and obstructed eccrine ducts [5]. In this case, since the mass began to develop after the patient had a circumcision, it was assumed that the circumcision was the cause of the mass. Penile epidermal cysts can be diagnosed by careful physical examination and radiological evaluation, including ultrasonography and computed tomography. Magnetic resonance imaging may be useful for cases suspected to have extension into the pelvis, although such cases are rare with only a few cases reported in the literature [5]. If a urethral diverticulum or a urethrocutaneous fistula is suspected, retrograde urethrography or voiding cystourethrography should be carried out [6]. On ultrasonography, epidermal cysts commonly appear as hypoechoic lesions with scattered echogenic reflectors. However, their appearances may vary according to the cyst's contents, ranging from an anechoic lesion to a hyperechoic, solid-appearing mass. Ruptured epidermal cysts are more likely to have lobulated contours and show color Doppler signals [3]. In this case, penile ultrasonography was performed and an increased echoic 
cystic mass was confirmed. As the boundary of the cyst was welldemarcated without invading the urethra or cavernous bodies, additional radiological evaluation was not performed. Indications for treatment of cysts are secondary cystic infection, pain upon intercourse, cosmetic reasons, and obstruction of the urinary tract. Simple complete excision followed by primary closure has generally been regarded as the best treatment procedure. Aspiration and simple drainage might carry a risk of recurrence [7]. It has been reported that re-excision is required for scrotal cysts when residual tissue is left after treatment [8]. In our case, because of the lasting pain, the large size, and the tendency to grow, the mass was totally excised under general anesthesia. The final histopathologic diagnosis was a ruptured epidermal cyst of the penis. In epidermal cysts requiring treatment, ruptured epidermal cysts appear at a low rate [9]. Such cysts are usually confirmed based on histological findings after surgery. Based on our experience, if a patient visits a hospital because of a slow-growing mass accompanied by sudden onset of penile pain, the possibility of a ruptured penile epidermal cyst should be considered, although it is rare.

\section{CONFLICT OF INTEREST}

No potential conflict of interest relevant to this article was reported.

\section{REFERENCES}

1. Erikci VS, Oney MD, Koyluoglu G. A rare complication after urethroplasty: epidermoid inclusion cyst. Clin Surg 2017;2:1671.

2. Chen HJ, Li W, Han YM, Che KY. A case of giant epidermoid cyst on the penis. Asian J Androl 2016;18:494-5.

3. Yang WT, Whitman GJ, Tse GM. Extratesticular epidermal cyst of the scrotum. AJR Am J Roentgenol 2004;183:1084.

4. Kumaraguru V, Prabhu R, Kannan NS. Penile epidermal cyst: a case report. J Clin Diagn Res 2016;10:PD05-6.

5. Guler Y, Tatar Z, Ucpinar B, Erbin A. An epidermal cyst of the penis after distal hypospadias surgery: a case report. J Med Case Rep 2018;12:378.

6. Jung JH, Eom M, Arkoncel FR, Sung YH, Kim W, Byun HK, et al. Penile epidermal cyst in a patient with augmentation penoplasty. Korean J Urol 2013;54:207-8.

7. Suwa M, Takeda M, Bilim V, Takahashi K. Epidermoid cyst of the penis: a case report and review of the literature. Int J Urol 2000;7:431-3.

8. Kalkan M, Sahih C, Ozyilmaz S, Ucmakli E. Multiple epidermoid cysts of penis. Eur J Gen Med 2012;9(Suppl 1):60-2.

9. Nigam JS, Bharti JN, Nair V, Gargade CB, Deshpande AH, Dey B, et al. Epidermal cysts: a clinicopathological analysis with emphasis on unusual findings. Int J Trichology 2017;9:108-12. 